\title{
Invasive giant pituitary macroadenoma causing Cushing disease; a case report
}

\author{
Gamage K K K ${ }^{1}$, Pathmanathan $\mathrm{S}^{1}$, Somasundaram $\mathrm{N} \mathrm{P}^{1}$, Sumanatilleke $\mathrm{M} \mathrm{R}^{1}$ \\ ${ }^{1}$ Diabetes and Endocrinology Unit. National Hospital of Sri Lanka
}

\section{Abstract}

Introduction: Cushing disease is a rare entity, and among them, giant pituitary adenomas causing Cushing disease is rarer. Here we present a case of Cushing disease secondary to a giant pituitary macroadenoma.

Case report: A 41-year-old female with poorly controlled diabetes mellitus and hypertension diagnosed for six months presented with a history of unintentional weight loss, polyuria and polydipsia for the same duration. There was change in her facial appearance with increased pigmentation, frontal balding and she also noted increase in the size of her abdomen. There were features of proximal muscle weakness. Symptoms of hypothyroidism, hypoadrenalism or hyperprolactinemia were absent, and she was amenorrhoeic following hysterectomy done a year back. Visual impairment, headache, abdominal pain, features suggestive of internal malignancy, especially lung and renal, including features of pheochromocytoma were absent. She denied a family history of similar illness.

Her BMI was $20.44 \mathrm{~kg} / \mathrm{m}^{-2}$. She had a prominent abdomen, round facies, hirsutism, male pattern hair loss, facial plethora and generalized pigmentation and thinning of skin. She also had hypertension and proximal muscle weakness. Rest of the examination was normal. Investigations revealed hypokalaemia, poorly controlled hyperglycaemia and, non-suppressed overnight dexamethasone suppression test. She had a elevated ACTH level, and a high dose dexamethasone suppression test showed suppression. Pituitary imaging with MRI revealed a 51x 62x $41 \mathrm{~mm}$ size sellar and a para sellar mass with heterogeneous echogenicity extending to the right temporal lobe. Further imaging for ectopic ACTH secretion was negative.

She underwent transcranial surgery which was unsuccessful due to intraoperative bleeding, and radiotherapy was given postoperatively.

Conclusion: Cushing disease due to giant pituitary macroadenomas are rare and may require secondary therapeutic measures in controlling the disease in case of failed surgery.

Keywords: Cushing disease, Giant pituitary adenoma, Invasive pituitary adenoma

Correspondence email: kavingakg@gmail.com

iD https://orcid.org/0000-0002-3943-7336

This is an open-access article distributed under the terms of the Creative Commons Attribution License, which permits unrestricted use, distribution, and reproduction in any medium, provided the original author and source are credited (CC BY 4.0) 


\section{Introduction}

Cushing disease accounts for a major portion of the cases of Cushing syndrome. Diagnosis and early initiation of treatment are challenging due to the caveats associated with the investigations. Cushing disease is less commonly due to macroadenomas. Giant pituitary adenomas, however causing Cushing disease is very rare. Here we present a case of Cushing disease due to a giant pituitary macroadenoma leading to severe hypercortisolism, with challenging the management issues in the resource-poor setting.

\section{Case report}

A 41-year-old Asian female, who was diagnosed with diabetes and hypertension six months before the current presentation, presented with a history of unintentional weight loss of $17 \mathrm{~kg}$ over six months period. She had polydipsia and polyuria, which awakes her 3-4 times in the night during sleep. With these symptoms, she was diagnosed with diabetes mellitus, and treatment had been started by a general practitioner. She had noted a gradual change in her appearance with increased skin pigmentation, swelling of the face, enlarging abdomen, excess growth of facial hair and significant hair loss with frontal balding, but there was no acne. She had features suggesting proximal muscle weakness, without episodes of muscle weakness or myalgia. Her glycaemic control had been poor with an $\mathrm{HbA} 1 \mathrm{c}$ of $11 \%$, despite increments of oral hypoglycaemic drugs and good adherence to medicine. There was no history of easy bruising, headache, visual impairment, seizures or abdominal pain. She did not have symptoms of hypothyroidism such as cold intolerance, constipation, and there was no history of galactorrhoea or, postural dizziness. She had undergone transabdominal hysterectomy at the age of 39 years for fibroid in the uterus when she had presented with menorrhagia. Until then, she had regular menstrual cycles. However, she had reduced libido during the preceding six months period. She found herself having a labile mood with frequent episodes of low mood, but without overt depressive symptoms or psychosis. There was no history of chronic cough, haemoptysis, haematuria, palpitations.

She was diagnosed with hypertension with a blood pressure reading of $170 / 100 \mathrm{mmHg}$. Similar to diabetes mellitus, there was poor blood pressure control despite being adherent to maximum tolerated doses of two antihypertensives. There is no history of bronchial asthma or exogenous steroid inhaler use. She denies abuse of steroids in the past as well as oral contraceptive use.

There was no family history of similar illness except for hypertension in her elder brother, and she is a product of non-consanguineous marriage. She has two children and is a housewife. She does not consume alcohol and is a non-smoker.

On examination, she had a prominent abdomen with wasted upper and lower limbs with a BMI of $20.44 \mathrm{kgm}^{-2}$. There was a generalized skin pigmentation. She had round facies, facial plethora, hirsutism, male pattern hair loss, but dorsal fat pad or supraclavicular fat pad was not prominent. She had thinning of skin and atrophic striae over the abdomen which were white in colour with a width of less than $1 \mathrm{~cm}$. There was no acne however extensive pityriasis versicolor infection over the chest and arms. Erythrasma was noted over the bilateral axillae. She did not have acanthosis nigricans. 
Cardiovascular system examination was normal except for the high blood pressure, which was $160 / 100 \mathrm{mmHg}$ on the initial presentation on both arms. Clinical evidence of left ventricular hypertrophy was absent. The abdominal and respiratory examination was normal. She had a GCS of 15/15; cranial nerve examination was normal without evidence of any visual field defects or evidence of hypertensive or diabetic retinopathy.

There was proximal more than distal wasting of the muscles of both upper and lower limbs with evidence of proximal weakness noted mainly in the lower limbs. Sensory examination was normal.

The initial evaluation consisted of the baseline investigations. Full blood count revealed a neutrophil leukocytosis (WBC 12.36x 103/microL; neutrophils $9.24 \mathrm{x}$ $10^{3} /$ microL) with normal haemoglobin level $(14.1 \mathrm{~g} / \mathrm{dL})$, and platelet counts (290x $10^{3} / \mathrm{microL}$ ). She had hypokalaemia (serum potassium $2.9 \mathrm{mmol} / \mathrm{L}$ ), with a normal sodium (142 mmol/L) and creatinine level $(0.48 \mathrm{mg} / \mathrm{dL})$. Liver functions were within the normal range. Capillary blood glucose on admission was $328 \mathrm{mg} / \mathrm{dL}$ with a HbA1c of $11.2 \%$.

Chest Xray was normal. Grade 2 fatty liver was present in the ultrasound scan abdomen; no intraabdominal masses were present, including suprarenal masses. Electrocardiogram and echocardiogram were normal, and no evidence of left ventricular hypertrophy was noted.

The endocrine evaluation was performed thereafter. Overnight dexamethasone suppression test (ODST) after $1 \mathrm{mg}$ of dexamethasone was non suppressed (1043.03 $\mathrm{nmol} / \mathrm{L})$. Low dose dexamethasone suppression test was done however due to sample collection error it could not be interpreted. Serum ACTH was elevated $(115 \mathrm{pg} / \mathrm{mL})$ suggesting ACTH dependent Cushing syndrome. High dose dexamethasone suppression test (HDDST) showed more than $50 \%$ suppression of the serum cortisol levels (baseline 9 am cortisol $1154.5 \mathrm{nmol} / \mathrm{L}$; after 48 hours 441.69 nmol/L). Imaging of the pituitary with Magnetic Resonance Imaging (MRI) revealed a 51x $62 \mathrm{x} 41 \mathrm{~mm}$ size sellar mass with heterogenous contents extending to suprasellar and bilateral para-sellar regions encasing right internal carotid artery (figure 1). There was an extension into the right middle cranial fossa compressing the temporal lobe. There was compression of the optic chiasma and optic tracts. Pituitary gland was not seen separately. There was no evidence of infarction or haemorrhage. Diagnosis o giant ACTH secreting pituitary adenoma was made. Rest of the pituitary hormonal evaluation revealed a serum prolactin of $159.01 \mathrm{mU} / \mathrm{L}$ (59-619), FSH $3.01 \mathrm{mIU} / \mathrm{mL}$ (21.7 - 153), LH 0.13 $\mathrm{mIU} / \mathrm{mL}$ (1.6-15), TSH $0.47 \mathrm{mIU} / \mathrm{L}$, Free T4 $\quad 1.32 \mathrm{ng} / \mathrm{dL} \quad(0.89-1.76)$. Serum testosterone $1.3 \mathrm{nmol} / \mathrm{L}(0.52-2.43)$. Serum corrected calcium was $2.4 \mathrm{mmol} / \mathrm{L}(2.1-2.5$ $\mathrm{mmol} / \mathrm{L})$.

Contrast-enhanced computed tomography (CECT) of chest and abdomen was normal without evidence of masses suggestive of ectopic ACTH secretion.

She was started on oral ketoconazole $200 \mathrm{mg}$ twice daily for management of hypercortisolism while monitoring liver enzymes which remained stable. Insulin was added for the management of hyperglycaemia. Oral potassium supplements along with spironolactone and losartan resulted in control of hypokalemia as well as 


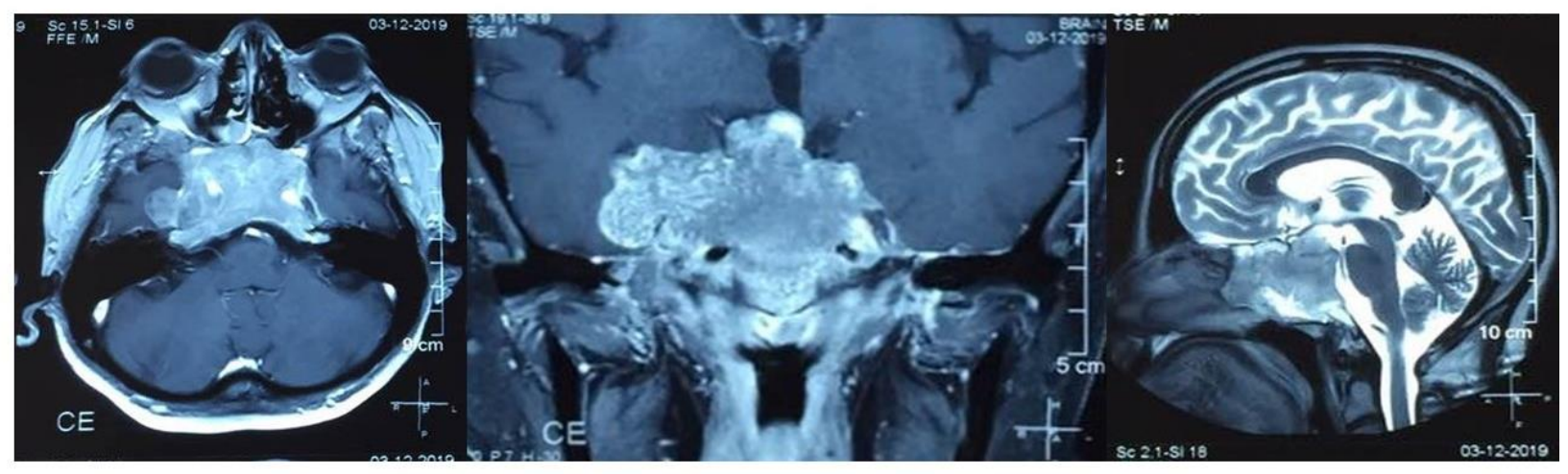

Figure 1: T1 and T2 weighted MRI showing the extensive tumour

hypertension. Due to poor control of hypercortisolism following initiation of ketoconazole she was started on intravenous etomidate infusion 1 week prior to surgery at a dose of $0.03 \mathrm{mg} / \mathrm{kg} /$ hour to control hypercortisolism in the preoperative period. A preoperative 9 am cortisol value of $375 \mathrm{nmol} / \mathrm{L}$ was achieved after 1 week of infusion. Surgery was unsuccessful due to bleeding, and only partial resection of the tumour was performed. Histopathology of the biopsy specimens showed multiple tumour tissue fragments composed of sheets and nests of monomorphic cells in a vascularized stroma. The constituent cells show uniform round nuclei with stippled chromatin and a moderate amount of basophilic cytoplasm and scattered areas with chromophobic cytoplasm. Mitotic activity is inconspicuous. There is no trabecular pattern or necrosis. The reticulin stain shows a disrupted pattern confirming the presence of an adenoma. Immunohistochemistry showed p53 wild type positivity. Ki67 proliferative index $<1 \%$. Tumour cells are negative for chromogranin, and diffuse cytoplasmic positivity is seen for pan CK (cytokeratin). ACTH staining was positive with dense granular pattern and with sparsely granulated cells. All these features were consistent with a pituitary adenoma. The cytokeratin pattern in the pituitary cell is suggestive of the type of hormone they secrete, where ACTH secreting cells tumour cells produce diffuse staining for cytokeratin ${ }^{(1)}$.

9 am cortisol on postoperative day 2 was still high 1012nmol/L. She was recommenced on ketoconazole for which she had poor compliance. Postoperative Cushing day curve while on ketoconazole $400 \mathrm{mg}$ daily resulted in a mean value of $540 \mathrm{nmol} / \mathrm{L}$ suggesting poorly controlled disease. She was referred for external beam radiotherapy after increasing the dose of ketoconazole from $200 \mathrm{mg}$ twice a day to $400 \mathrm{mg}$ twice a day which improved hypokalemia, hypertension as well as hyperglycemia.

\section{Discussion}

ACTH secreting pituitary adenomas are known to have a lower prognosis when it comes to large size. Generally, for macroadenomas, it ranges from 12.5$71.1 \%{ }^{(2)}$. However, with the inadequate tumour resection in this patient, the expected prognosis is low.

The Endocrine Society guideline recommends positivity of at least two urinary free cortisol levels, an overnight dexamethasone suppression test, at least two 
late-night salivary cortisol levels or low dose dexamethasone suppression test for diagnosis of hypercortisolism once exogenous cortisol intake is excluded ${ }^{(3)}$. Recent use of oral contraceptive pills was excluded to avoid confusion with high serum cortisol levels due to high cortisol binding globulin $^{(4)}$. As the test was done in the hospital, it was ensured the correct doe of dexamethasone was taken at the correct timing. Medicines that would have caused increased metabolism of the given dexamethasone was also excluded out of which the commonly used medications are rifampicin, carbamazepine, phenytoin and phenobarbital ${ }^{(5)}$. The sensitivity of ODST is around $94-100 \%$, with a specificity of around $87.5 \%{ }^{(5)}$. The reported cut-off values for ODST was around $100-200 \mathrm{nmol} / \mathrm{L}$, however lower cut-off of $50 \mathrm{nmol} / \mathrm{L}$ is used as it excludes Cushing syndrome at an outpatient setting easily even though it has higher false-positive values ${ }^{(6)}$. On the other hand, the low dose dexamethasone suppression test, which also uses a cut-off of $50 \mathrm{nmol} / \mathrm{L}$ for serum cortisol after a 48 -hour suppression with a total of $2 \mathrm{mg}$ of dexamethasone, has a sensitivity and specificity of $97-100 \%{ }^{(6)}$. However, this was not carried out as it takes a longer duration. The advantage of LDDST here would have also been in accordance to its higher sensitivity when compared to patients with pseudo-Cushing is that this had a sensitivity of $90 \%$ and a specificity of $100 \%$ when diagnosing Cushing syndrome ${ }^{(6)}$. Later studies demonstrated more reduced specificity and sensitivity ${ }^{(3)}$. LDDST in our patient was not interpreted due to error in sample collection. As the patient had overt features of Cushing and ODST values of more than $1000 \mathrm{nmol} / \mathrm{L}$ therefore further evaluation was carried out to find the focus of hypercortisolism.
Caveats in HDDST is that about $10 \%$ of the patients with ectopic ACTH secretion can have a positive response with suppression by dexamethasone. Also, $90 \%$ of the patients with Cushing disease show suppression also about $50 \%$ of the patients with ectopic ACTH secretion due to carcinoids syndromes $^{(7)}$ therefore it does not provide additional information compared to the MRI pituitary or inferior petrosal sinus sampling. However, in our patient HDDST was suppressed less than 50\% supporting the possibility of pituitary dependent ACTH secretion.

Only $4-10 \%$ of ACTH secreting pituitary tumours are pituitary macroadenomas ${ }^{(8)}$. Rest are microadenomas. Therefore surgery makes them cured once performed and has a success rate of $90 \%$ when performed at a high volume centre $^{(9)}$. Transsphenoidal hypophysectomy often is adequate, which ideally to be done by enucleation of the tumour alone. For those who fail such surgery, other treatment options are total hypophysectomy, stereotactic or conventional external beam radiotherapy, medical management with adrenolytic medications or bilateral adrenalectomy. To avoid Nelson syndrome will need radiotherapy $^{(9)}$.

Pituitary macroadenomas are considered as 'giant' when they exceed the size of $4 \mathrm{~cm}$. The behaviour of ACTH secreting macroadenomas differ from microadenomas as they tend to secrete higher levels of ACTH as well as cortisol compared to microadenomas. Also, the use of HDDST is not reliable in differentiating from ectopic tumours, as they also tend to be nonsuppressible $^{(8)}$. However, our patient had suppressed values. 
According to the available guideline sources the presence of an adenoma of $>6 \mathrm{~mm}$ size is suggestive of the pituitary origin of ACTH secretion therefore routine use of inferior petrosal sinus sampling is not routinely recommended in such state ${ }^{(7)}$. Therefore, it was not carried out in our patient. Further imaging with CECT chest, abdomen and pelvis also confirmed the absence of ectopic tumours.

In general, invasive tumours are associated with a higher Ki 67 index which was low in our patient. But there was wild type positivity of p53 in the tumour. Expression of p53 is associated with tumours which have a higher proliferative status ${ }^{(10)}$. Thus, close follow up is required in this patient as the risk of tumour progression may occur. In such case, bilateral adrenalectomy needs to be considered. Redo surgery was of at high risk according to the surgical team due to increased risk of bleeding in to the tumour, for which the consent was not given by the patient after explanation. In general, it takes around six months to one year for the external beam radiotherapy to work so she will require to continue the steroidogenic inhibitors. Close monitoring of liver functions is required for ketoconazole and monitoring of cortisol levels as when the patients tumour starts to respond to radiotherapy there will be hypoadrenalism as well. Reduced cortisol synthesis in turn will increase ACTH secretion and initial improvement in control of hypercortisolism may disappear due to increasing production due to ACTH. In one study, this was shown to occur after long durations of therapy, more than 24 months, and was shown to occur in about $15 \%$ of patients ${ }^{(11)}$.

Furthermore, until the patient is cured, should explain the complications of the disease, including thromboembolic risk and risk of infections, including opportunistic infections such as pneumocystis jirovecii pneumonia. With the improvement of hypercortisolism, patients should be warned that they may experience hypoglycemia and careful monitoring of serum potassium with tailing off aldosterone antagonists will be required.

Cushing disease is known to have a benign disease course. However, active Cushing disease has a higher rate of mortality compared to the normal population, which increases by four-fold, mainly due to cardiovascular disease ${ }^{(12)}$. Older age at diagnosis, male sex and preoperative high plasma ACTH concentration is associated with a higher rate of mortality ${ }^{(12)}$.

\section{Conclusion}

In conclusion, rarely Cushing disease can present as invasive pituitary macroadenoma and may challenge the management due to being unamenable for surgery.

\section{List of abbreviations}

ACTH: Adrenocorticotrophic Hormone

LDDST: Low dose dexamethasone suppression test

HDDST: High dose dexamethasone suppression test

GCS: Glasgow coma scale

CECT: Contrast enhanced computed tomography

MRI: Magnetic Resonance Imaging

\section{Declarations}

- Ethics approval and consent to participate- Written informed consent was obtained from the patient for publication of this case report and for accompanying images. A copy of the written consent is available for review by the Editor-in- 
Chief of this journal.

- Consent for publication- Written informed consent was obtained from the patient for publication of this case report and for accompanying images. A copy of the written consent is available for review by the Editor-in-Chief of this journal

Availability of data and materials: available to be reviewed by the Editor-in- chief

\section{Conflict of interest}

None

Funding

None

\section{Authors' contributions}

KKKG is the first author and managed the patient. MRS and NPS, SP participated in managing the patient and supported manuscript writing. All authors have read and approved the manuscript, and ensure that this is the case.

\section{Acknowledgements}

None

\section{References}

1. Höfler H, Denk H, Walter GF. Immunohistochemical demonstration of cytokeratins in endocrine cells of the human pituitary gland and in pituitary adenomas. Virchows Arch A Pathol Anat Histopathol. 1984;404(4):359-68. doi: 10.1007/BF00695220. PMID: 6208678.

2. Zhuang Z, Liu X, Bao X, Pan B, Deng K, Yao Y, Lian W, Xing B, Zhu H, Lu L, Wang R, Feng M. Invasive ACTH-secreting pituitary macroadenoma in remission after transsphenoidal resection: A case report and literature review. Medicine (Baltimore). 2018
Nov;97(46):e13148.doi: 10.1097/MD.

0000000000013148.PMID: 30431585; PMCID: PMC6257388.

3. Lynnette K. Nieman, Beverly M. K. Biller, James W. Findling, John Newell-Price, Martin O. Savage, Paul M. Stewart, Victor M. Montori, The Diagnosis of Cushing's Syndrome: An Endocrine Society Clinical Practice Guideline, The Journal of Clinical Endocrinology \& Metabolism, 2008 May;93(5):1526-1540,https://doi.org /10. 1210 /jc.2008-0125

4. Dommisse CS, Hayes PE, Kwentus JA. Effect of estrogens on the dexamethasone suppression test in nondepressed women. $J$ Clin Psychopharmacol. 1985 Dec;5(6):315-9. PMID: 4066996.

5. Bansal V, El Asmar N, Selman WR, Arafah BM. Pitfalls in the diagnosis and management of Cushing's syndrome. Neurosurg Focus. 2015 Feb;38(2):E4.

doi:

10.3171/2014.11.FOCUS14704.

PMID:

25639322.

6. Newell-Price J, Trainer P, Besser M, Grossman A. The diagnosis and differential diagnosis of Cushing's syndrome and pseudo-Cushing's states. Endocr Rev. 1998 Oct;19(5):647-72. doi: 10.1210/edrv.19.5.0346. PMID: 9793762.

7. Somasundaram N, Rajaratnam $\mathrm{H}$, Wijeyarathne C, Bulugahapitiya U, Siyambalapitiya S, Antonypillai C, Dr. Garusinghe C, Muthukuda D, Niranjala M. W. S, Karunasena N, Swarnasri W. S. T., Aravinthan M., Kottahachchi D. C., Ranasinghe L.D. Cushing syndrome, Clinical guideline, Endocrine Society of Sri Lanka. 2014;

8. Woo YS, Isidori AM, Wat WZ, Kaltsas GA, Afshar F, Sabin I, Jenkins PJ, Monson JP, Besser GM, Grossman AB. Clinical and biochemical characteristics of adrenocorticotropin-secreting macro adenomas. J Clin Endocrinol Metab. 2005 Aug;90(8):4963-9. doi: 10.1210/jc.2005-0070. Epub 2005 May 10. PMID: 15886242. 
9. Kelly DF. Transsphenoidal surgery for Cushing's disease: a review of success rates, remission predictors, management of failed surgery, and Nelson's Syndrome. Neurosurg Focus. 2007;23(3):E5. doi: 10.3171/ foc.2007.23.3.7. PMID: 17961026.

10. Suliman M, Royds J, Cullen D, Timperley W, Powell T, Battersby R, Jones TH. Mdm2 and the p53 pathway in human pituitary adenomas. Clin Endocrinol (Oxf). 2001 Mar;54(3):317-25. doi: 10.1046/j.1365-2265.2001.01195.x. PMID: 11298083.

11. Castinetti F, Guignat L, Giraud P, Muller M, Kamenicky P, Drui D, Caron P, Luca F, Donadille B, Vantyghem MC, Bihan H, Delemer B, Raverot G, Motte E, Philippon M, Morange I,
Conte-Devolx B, Quinquis L, Martinie M, Vezzosi D, Le Bras M, Baudry C, Christin-Maitre S, Goichot B, Chanson P, Young J, Chabre O, Tabarin A, Bertherat J, Brue T. Ketoconazole in Cushing's disease: is it worth a try? J Clin Endocrinol Metab. 2014 May;99(5):1623-30. doi: 10.1210/jc.2013-3628. Epub 2014 Jan 28. PMID: 24471573.

12. Lambert JK, Goldberg L, Fayngold S, Kostadinov J, Post KD, Geer EB. Predictors of mortality and long-term outcomes in treated Cushing's disease: a study of 346 patients. J Clin Endocrinol Metab. 2013 Mar;98(3):1022-30. doi: 10.1210/jc.2012-2893. Epub 2013 Feb 7. PMID: 23393167; PMCID: PMC3590483. 